\title{
HOMOLOGICAL DIMENSIONS AND APPROXIMATE CONTRACTIBILITY FOR KÖTHE ALGEBRAS
}

\author{
ALEXEI YU. PIRKOVSKII \\ Department of Geometry and Topology, Faculty of Mathematics \\ State University - Higher School of Economics \\ Vavilova 7, 117312 Moscow, Russia \\ E-mail: aupirkovskii@hse.ru,pirkosha@online.ru
}

\begin{abstract}
We give a survey of our recent results on homological properties of Köthe algebras, with an emphasis on biprojectivity, biflatness, and homological dimension. Some new results on the approximate contractibility of Köthe algebras are also presented.
\end{abstract}

1. Introduction. From the Banach algebra theory point of view, Köthe algebras are weighted locally convex analogues of $\ell^{1}$ with pointwise multiplication. The underlying locally convex spaces of Köthe algebras (Köthe sequence spaces) are classical objects, which have been studied since the 1940's 15 and are often used to provide various examples and counterexamples in the theory of topological vector spaces. The idea to consider Köthe spaces as algebras under pointwise multiplication is apparently due to S. J. Bhatt and G. M. Deheri [1. The study of homological properties of Köthe algebras was started by the author 21 .

The present paper is organized as follows. In Sections 2 and 3, we recall the definitions and give some basic examples of Köthe spaces and Köthe algebras. In Section 4, we give a brief outline of Topological Homology, i.e., the homology theory for topological algebras. In Section 5, we introduce and discuss four conditions (denoted $(\mathbf{U}),(\mathbf{N}),(\mathbf{B})$, and $(\mathbf{M})$ ) on Köthe sets. These conditions are then applied to computing homological dimensions of Köthe algebras in Section 6. A detailed exposition of the results of Sections 5 and 6 (including proofs) can be found in $21,22,22,24$. Section 7 contains some new results on the approximate contractibility of Köthe algebras. Among other things, we show that a nuclear biprojective Köthe algebra is approximately contractible. Finally, in Section 8

2010 Mathematics Subject Classification: Primary 46M18, 46H25, 16E10; Secondary 46A45, $16 \mathrm{D} 40,18 \mathrm{G} 25$.

Key words and phrases: Köthe algebra, (weak) global dimension, (weak) bidimension, approximately contractible Fréchet algebra.

The paper is in final form and no version of it will be published elsewhere. 
we formulate some open problems related to homological properties of Fréchet algebras and, in particular, of Köthe algebras.

2. Köthe spaces. Let $I$ be any set, and let $P$ be a set of nonnegative real-valued functions on $I$. For $p \in P$, we will write $p_{i}$ for $p(i)$. Recall that $P$ is a Köthe set on $I$ if the following axioms are satisfied:

$$
\begin{aligned}
& \forall i \in I \quad \exists p \in P: \quad p_{i}>0 ; \\
& \forall p, q \in I \quad \exists r \in P: \quad \max \left\{p_{i}, q_{i}\right\} \leq r_{i} \quad \forall i \in I .
\end{aligned}
$$

Given a Köthe set $P$, the Köthe space $\lambda(P)$ is defined as follows:

$$
\lambda(P)=\left\{x=\left(x_{i}\right) \in \mathbb{C}^{I}:\|x\|_{p}=\sum_{i}\left|x_{i}\right| p_{i}<\infty \quad \forall p \in P\right\} .
$$

This is a complete locally convex space with the topology determined by the family of seminorms $\left\{\|\cdot\|_{p}: p \in P\right\}$. Clearly, $\lambda(P)$ is a Fréchet space if and only if $P$ contains an at most countable cofinal subset.

Convention. To be definite, we will always assume that $P$ is countable, although some of our results hold without this assumption.

Let us give some standard examples of Köthe spaces. In all these examples, we set $I=\mathbb{N}$, so that elements of $P$ and of $\lambda(P)$ are sequences.

ExAmple 2.1. If $P$ consists of only one sequence $(1,1, \ldots)$, then $\lambda(P)=\ell^{1}$.

EXAmple 2.2. For each $n \in \mathbb{N}$ set $p^{(n)}=(1, \ldots, 1,0, \ldots)$ with 1 repeated $n$ times, and let $P=\left\{p^{(n)}: n \in \mathbb{N}\right\}$. Then $\lambda(P)=\mathbb{C}^{\mathbb{N}}$, the space of all complex sequences with the topology of pointwise convergence. More generally, if $I$ is any set and $P$ is the family of all nonnegative functions on $I$ with finite support, then $\lambda(P)=\mathbb{C}^{I}$.

EXAMPLE 2.3. For each $n \in \mathbb{N}$ set $p^{(n)}=\left(1^{n}, 2^{n}, \ldots, k^{n}, \ldots\right)$, and let $P=\left\{p^{(n)}\right.$ : $n \in \mathbb{N}\}$. The resulting Köthe space $\lambda(P)$ is denoted by $s$ and is called the space of rapidly decreasing sequences.

ExAmple 2.4. For each $n \in \mathbb{N}$ set $p^{(n)}=\left(n^{1}, n^{2}, \ldots, n^{k}, \ldots\right)$, and let $P=\left\{p^{(n)}: n \in \mathbb{N}\right\}$. It is easy to show that the resulting Köthe space $\lambda(P)$ is topologically isomorphic to the space $\mathscr{O}(\mathbb{C})$ of entire functions with the topology of compact convergence. Explicitly, the isomorphism $\mathscr{O}(\mathbb{C}) \rightarrow \lambda(P)$ takes each entire function to the sequence of its Taylor coefficients at 0 .

ExAmple 2.5. This example generalizes Examples 2.3 and 2.4 Fix a real number $0<$ $R \leq \infty$ and a nondecreasing sequence $\alpha=\left(\alpha_{n}\right)_{n \in \mathbb{N}}$ of positive numbers with $\lim _{n} \alpha_{n}=$ $\infty$. Let $P=\left\{\left(r^{\alpha_{k}}\right)_{k \in \mathbb{N}}: 0<r<R\right\}$. The resulting Köthe space $\lambda(P)$ is denoted by $\Lambda_{R}(\alpha)$ and is called a power series space (of finite type if $R<\infty$, and of infinite type if $R=\infty)$. Of course, the above Köthe set $P$ is uncountable, but we can easily make it countable (e.g., by considering only rational $r$ ) without changing $\lambda(P)$.

It is easy to see that if $\alpha_{n}=\log n$, then $\Lambda_{\infty}(\alpha)=s$ (see Example 2.3). If $\alpha_{n}=n$, then $\Lambda_{R}(\alpha)$ is topologically isomorphic to $\mathscr{O}\left(\mathbb{D}_{R}\right)$, the space of holomorphic functions on the disk $\mathbb{D}_{R}=\{z \in \mathbb{C}:|z|<R\}$ (see Example 2.4). 
3. Köthe algebras. Throughout, all vector spaces and algebras are assumed to be over the field $\mathbb{C}$ of complex numbers. All algebras are assumed to be associative, but not necessarily unital. The unitization of an algebra $A$ is denoted by $A_{+}$. By a Fréchet algebra we mean a complete metrizable locally convex algebra (i.e., a topological algebra whose underlying space is a Fréchet space). A locally $m$-convex algebra is a topological algebra $A$ whose topology can be defined by a family of submultiplicative seminorms (i.e., seminorms $\|\cdot\|$ satisfying $\|a b\| \leq\|a\|\|b\|$ for all $a, b \in A)$. Note that, unlike some authors, we do not require Fréchet algebras to be locally $m$-convex.

Given a set $I, \mathbb{C}^{I}$ is clearly an algebra under pointwise multiplication. It is natural to ask when $\lambda(P)$ is a subalgebra of $\mathbb{C}^{I}$, and when it is a Fréchet algebra (under its canonical topology). To answer these questions, let us introduce some notation.

Notation. Let $P, Q$ be Köthe sets on $I$. We say that $P$ is dominated by $Q$ and write $P \prec Q$ if for each $p \in P$ there exist $q \in Q$ and $C>0$ such that $p_{i} \leq C q_{i}$ for all $i \in I$ (we write $p \leq C q$ for brevity). This is equivalent to say that $\lambda(Q) \subset \lambda(P)$, and the embedding of $\lambda(Q)$ into $\lambda(P)$ is continuous. In fact, since $P$ and $Q$ are assumed to be countable, it is easy to show that $P \prec Q$ if and only if $\lambda(Q) \subset \lambda(P)$ (the continuity of the embedding will then hold automatically). If $P \prec Q$ and $Q \prec P$, then we say that $P$ and $Q$ are equivalent and write $P \sim Q$. This means exactly that $\lambda(P)=\lambda(Q)$ topologically, or, equivalently, that $\lambda(P)=\lambda(Q)$ as sets. The product $P \cdot Q$ is defined to be the Köthe set consisting of all functions $p q=\left(p_{i} q_{i}\right)_{i \in I}$, where $p \in P$ and $q \in Q$. Finally, we let $P^{2}=P \cdot P$, which is equivalent to the Köthe set $\left\{p^{2}=\left(p_{i}^{2}\right)_{i \in I}: p \in P\right\}$.

Proposition 3.1. For a Köthe set $P$, the following conditions are equivalent:

(i) $\lambda(P)$ is a subalgebra of $\mathbb{C}^{I}$;

(ii) $\lambda(P)$ is a subalgebra of $\mathbb{C}^{I}$ and is a Fréchet algebra under its canonical topology;

(iii) $P \prec P^{2}$.

Proof. (i) $\Rightarrow$ (iii). Since $P$ is countable, we may assume that $P=\left\{p^{(k)}: k \in \mathbb{N}\right\}$ and that $p^{(k)} \leq p^{(k+1)}$ for all $k$. Assume, towards a contradiction, that $P$ is not dominated by $P^{2}$. Then there exists $m \in \mathbb{N}$ such that there are no $k \in \mathbb{N}$ and $C>0$ satisfying $p^{(m)} \leq C\left(p^{(k)}\right)^{2}$. Without loss of generality, we may assume that $m=1$. Then for each $k \in \mathbb{N}$ there exists $i_{k} \in I$ such that

$$
p_{i_{k}}^{(1)}>k^{4}\left(p_{i_{k}}^{(k)}\right)^{2} .
$$

We may also assume that $i_{k} \neq i_{l}$ for $k \neq l$. Note that (1) implies that $p_{i_{k}}^{(1)}>0$ and hence $p_{i_{k}}^{(k)}>0$ for all $k$. Now define a function $x=\left(x_{i}\right)_{i \in I}$ on $I$ by

$$
x_{i}= \begin{cases}\frac{1}{k^{2} p_{i_{k}}^{(k)}} & \text { if } i=i_{k}, \\ 0 & \text { if } i \notin\left\{i_{1}, i_{2}, \ldots\right\} .\end{cases}
$$

For each $l \in \mathbb{N}$ we have

$$
\sum_{k \geq l}\left|x_{i_{k}}\right| p_{i_{k}}^{(l)} \leq \sum_{k \geq l}\left|x_{i_{k}}\right| p_{i_{k}}^{(k)}=\sum_{k \geq l} \frac{1}{k^{2}}<\infty
$$


so that $x \in \lambda(P)$. On the other hand, (1) implies that

$$
\sum_{i}\left|x_{i}^{2}\right| p_{i}^{(1)}=\sum_{k}\left|x_{i_{k}}^{2}\right| p_{i_{k}}^{(1)}=\sum_{k} \frac{p_{i_{k}}^{(1)}}{k^{4}\left(p_{i_{k}}^{(k)}\right)^{2}}=\infty,
$$

i.e., $x^{2} \notin \lambda(P)$. Thus $\lambda(P)$ is not a subalgebra of $\mathbb{C}^{I}$, which contradicts (i).

(iii) $\Rightarrow$ (ii). Given $p \in P$, find $q \in P$ and $C>0$ such that $p \leq C q^{2}$. Then for each $a, b \in \lambda(P)$ we have

$$
\|a b\|_{p}=\sum_{i}\left|a_{i} b_{i}\right| p_{i} \leq C \sum_{i}\left|a_{i}\right|\left\|b_{i} \mid q_{i}^{2} \leq C\right\| a\left\|_{q}\right\| b \|_{q}<\infty .
$$

Therefore $a b \in \lambda(P)$, i.e., $\lambda(P)$ is a subalgebra of $\mathbb{C}^{I}$. The continuity of the multiplication on $\lambda(P)$ is immediate from 2 .

(ii) $\Rightarrow$ (i). This is clear.

REMARK 3.2. If $P$ is arbitrary (i.e., not necessarily countable), then condition (iii) is equivalent to the assertion that $\lambda(P)$ is a subalgebra of $\mathbb{C}^{I}$ and is a topological algebra with jointly continuous multiplication.

Definition 3.3. Algebras of the form $\lambda(P)$ (where $P$ is a Köthe set satisfying $P \prec P^{2}$ ) are called Köthe algebras.

REMARK 3.4. In many natural cases (see examples below), we have $p_{i} \in\{0\} \cup[1,+\infty$ ) for all $p \in P, i \in I$. This implies that $p_{i} \leq p_{i}^{2}$, so that $P \prec P^{2}$, and $\lambda(P)$ is a Köthe algebra. Moreover, the seminorms $\|\cdot\|_{p}$ are submultiplicative in this case, and so $\lambda(P)$ is locally $m$-convex.

Examples 3.5. Clearly, $\ell^{1}$ and $\mathbb{C}^{I}$ are Köthe algebras. The power series space $\Lambda_{R}(\alpha)$ is a Köthe algebra if and only if $R \geq 1$. Moreover, if $R>1$, then $\Lambda_{R}(\alpha)$ is locally $m$-convex. In particular, identifying the space $\mathscr{O}\left(\mathbb{D}_{R}\right)$ (for $R \geq 1$ ) with $\Lambda_{R}(\{n\})$ (see Example 2.5), we see that $\mathscr{O}\left(\mathbb{D}_{R}\right)$ becomes a Fréchet algebra under the "componentwise" product of the Taylor expansions of holomorphic functions (the Hadamard product; see [25]). The resulting topological algebra is denoted by $\mathscr{H}\left(\mathbb{D}_{R}\right)$ and is called the Hadamard algebra.

4. Topological Homology. Now let us briefly recall some basic notions of Topological Homology, i.e., the homology theory for locally convex topological algebras. This theory was developed in the early 1970's by A. Ya. Helemskii (see, e.g., [8]) in the special case of Banach algebras. A few years later a similar theory was independently discovered by R. Kiehl and J. L. Verdier [14] and by J. L. Taylor [27] in the context of more general topological algebras. For details, we refer to Helemskii's monograph [10.

To be definite, we will work only with Fréchet modules over Fréchet algebras. Let $A$ be a Fréchet algebra. A left Fréchet $A$-module is a left $A$-module $X$ endowed with a Fréchet space topology in such a way that the action $A \times X \rightarrow X$ is continuous. Left Fréchet $A$-modules and their continuous morphisms form a category denoted by $A$-mod. Given $X, Y \in A$-mod, the space of morphisms from $X$ to $Y$ will be denoted by $\mathbf{h}_{A}(X, Y)$. The categories mod- $A$ and $A$-mod- $A$ of right Fréchet $A$-modules and of Fréchet $A$-bimodules are defined similarly. 
If $X$ is a right Fréchet $A$-module and $Y$ is a left Fréchet $A$-module, then their $A$-module tensor product $X \widehat{\otimes}_{A} Y$ is defined to be the quotient $(X \widehat{\otimes} Y) / N$, where $N \subset X \widehat{\otimes} Y$ is the closed linear span of all elements of the form $x \cdot a \otimes y-x \otimes a \cdot y(x \in X, y \in Y, a \in A)$. As in pure algebra, the $A$-module tensor product can be characterized by the universal property that, for each Fréchet space $E$, there is a natural bijection between the set of all continuous $A$-balanced bilinear maps from $X \times Y$ to $E$ and the set of all continuous linear maps from $X \widehat{\otimes}_{A} Y$ to $E$.

A chain complex $C_{\bullet}=\left(C_{n}, d_{n}\right)_{n \in \mathbb{Z}}$ in $A$-mod is admissible if it splits in the category of topological vector spaces, i.e., if it has a contracting homotopy consisting of continuous linear maps. Geometrically, this means that $C_{\bullet}$ is exact, and $\operatorname{Ker} d_{n}$ is a complemented subspace of $C_{n}$ for each $n$.

Let Vect denote the category of vector spaces and linear maps. A left Fréchet $A$-module $P$ is projective if the functor $\mathbf{h}_{A}(P,-): A$-mod $\rightarrow$ Vect is exact in the sense that it takes admissible sequences of Fréchet $A$-modules to exact sequences of vector spaces. A left Fréchet $A$-module $F$ is flat if the tensor product functor $(-) \widehat{\otimes}_{A} F: \bmod -A \rightarrow$ Vect is exact in the same sense as above. It is known that every projective Fréchet module is flat.

A resolution of $X \in A$-mod is a pair $\left(P_{\bullet}, \varepsilon\right)$ consisting of a nonnegative chain complex $P_{\bullet}$ in $A$-mod and a morphism $\varepsilon: P_{0} \rightarrow X$ making the sequence $P_{\bullet} \stackrel{\varepsilon}{\rightarrow} X \rightarrow 0$ into an admissible complex. The length of $P_{\boldsymbol{\bullet}}$ is the minimum integer $n$ such that $P_{i}=0$ for all $i>n$, or $\infty$ if there is no such $n$. If all the $P_{i}$ 's are projective (respectively, flat), then $(P \bullet, \varepsilon)$ is called a projective resolution (respectively, a flat resolution) of $X$. It is a standard fact that $A$-mod has enough projectives, i.e., each left Fréchet $A$-module has a projective resolution. The same is true of $\bmod -A$ and $A-\bmod -A$.

The projective homological dimension of $X \in A$-mod is the minimum integer $n=$ $\operatorname{dh}_{A} X \in \mathbb{Z}_{+} \cup\{\infty\}$ with the property that $X$ has a projective resolution of length $n$. Similarly, the weak homological dimension of $X \in A$-mod is the minimum integer $n=$ w.dh ${ }_{A} X \in \mathbb{Z}_{+} \cup\{\infty\}$ with the property that $X$ has a flat resolution of length $n$. Heuristically, the projective (respectively, flat) dimension measures how far $X$ is from being projective (respectively, flat). In particular, $\mathrm{dh}_{A} X=0$ if and only if $X$ is projective, and w. $\mathrm{dh}_{A} X=0$ if and only if $X$ is flat. Since each projective module is flat, we clearly have w. $\operatorname{dh}_{A} X \leq \operatorname{dh}_{A} X$.

Given a Fréchet algebra $A$, the global dimension and the weak global dimension of $A$ are defined by

$$
\operatorname{dg} A=\sup \left\{\operatorname{dh}_{A} X \mid X \in A \text {-mod }\right\}, \quad \text { w.dg } A=\sup \left\{\mathrm{w} \cdot \operatorname{dh}_{A} X \mid X \in A \text {-mod }\right\} .
$$

The bidimension and the weak bidimension of $A$ are defined by

$$
\mathrm{db} A=\mathrm{dh}_{A-A} A_{+}, \quad \text { w.db } A=\mathrm{w} \cdot \mathrm{dh}_{A-A} A_{+},
$$

where the subscript " $A$ - $A$ " means that we work in the category $A$-mod- $A$. We clearly have w.dg $A \leq \operatorname{dg} A$ and w.db $A \leq \operatorname{db} A$. It is also true (but less obvious) that $\operatorname{dg} A \leq \operatorname{db} A$ and w.dg $A \leq$ w.db $A$.

To complete our short introduction to Topological Homology, let us recall some "homological triviality" conditions for Fréchet algebras. By definition, a Fréchet algebra $A$ 
is biprojective (respectively, biflat) if $A$ is projective (respectively, flat) in $A$-mod- $A$. Similarly, $A$ is contractible (respectively, amenable) if $A_{+}$is projective (respectively, flat) in $A$-mod- $A$. In fact, there are many other equivalent definitions of the above notions. For example, it is immediate from the definition of $\mathrm{db}$ that $A$ is contractible if and only if $\operatorname{db} A=0$. This is equivalent to say that for each Fréchet $A$-bimodule $X$ all continuous derivations from $A$ to $X$ are inner (see [11, 7.3.37]). Similarly, $A$ is amenable if and only if w.db $A=0$, which is equivalent to say that for each Fréchet $A$-bimodule $X$ all continuous derivations from $A$ to $X^{*}$ (where $X^{*}$ is the strong dual of $X$ ) are inner (see [11, 7.3.37] for the Banach algebra case and [23, Corollary 3.5] for the Fréchet algebra case). In fact, the above characterization of amenability of Banach algebras in terms of derivations was the original definition of amenability due to B. E. Johnson [13.

Let us also remark that a Fréchet algebra $A$ is biprojective if and only if the product map $A \widehat{\otimes} A \rightarrow A$ is a retraction in $A$-mod- $A$ [10, IV.5.6]. There is also a dual characterization of biflat Banach algebras [10, VII.2.7], but we will not use it. Recall also that a Fréchet algebra $A$ is contractible if and only if $A$ is biprojective and unital [10, IV.5.8].

The following theorem is essentially due to Helemskii [10, V.2.28], [12, 2.5.8]; see also [23. Proposition 4.8] for the Fréchet algebra case.

TheOrem. Let $A$ be a Fréchet algebra.

(i) If $A$ is biprojective, then $\mathrm{db} A \leq 2$.

(ii) If $A$ is biflat, then w.dg $A \leq 2$.

(iii) If $A$ is biflat and projective in $A$-mod or in $\bmod -A$, then $\mathrm{w} . \mathrm{db} A \leq 2$.

(iv) If $A$ is a biflat Banach algebra, then w.db $A \leq 2$.

We do not know whether part (iv) of the above theorem holds for biflat Fréchet algebras.

5. Some conditions on Köthe sets. Our task is to compute the homological dimensions dg, db, w.dg, and w.db of Köthe algebras. To this end, let us introduce some conditions on Köthe sets.

Let $\lambda(P)$ be a Köthe algebra.

Definition 5.1. We say that $P$ satisfies $(\mathbf{U})$ if

$$
\sum_{i} p_{i}<\infty \text { for all } p \in P .
$$

Clearly, $P$ satisfies $(\mathbf{U})$ if and only if $\lambda(P)$ is unital (whence the notation " $(\mathbf{U})$ ").

EXAMPLE 5.2. $\mathbb{C}^{I}$ is unital.

ExAmple 5.3. The Hadamard algebra $\mathscr{H}\left(\mathbb{D}_{1}\right)$ is unital. The identity element of $\mathscr{H}\left(\mathbb{D}_{1}\right)$ is the function $f(z)=(1-z)^{-1}$, whose Taylor coefficients are all equal to 1 .

EXAMPLE 5.4. The algebra $\Lambda_{R}(\alpha)$ is not unital unless $R=1$. In particular, the algebra $s$ of rapidly decreasing sequences and the Hadamard algebras $\mathscr{H}\left(\mathbb{D}_{R}\right)$ for $R>1$ are not unital. 
Definition 5.5. We say that $P$ satisfies $(\mathbf{N})$ if

$$
\forall p \in P \quad \exists q \in P \quad \exists \alpha \in \ell^{1}(I): \quad p \leq \alpha q .
$$

The Grothendieck-Pietsch criterion (see [20,6.1.2]) states that $P$ satisfies $(\mathbf{N})$ if and only if $\lambda(P)$ is nuclear (whence the notation "(N)").

There is another useful form of the Grothendieck-Pietsch criterion. Given a Köthe set $P$, let

$$
\lambda^{\infty}(P)=\left\{a=\left(a_{i}\right) \in \mathbb{C}^{I}:\|a\|_{p}^{\infty}=\sup _{i}\left|a_{i}\right| p_{i}<\infty \quad \forall p \in P\right\} .
$$

This is a complete locally convex space with the topology determined by the family of seminorms $\left\{\|\cdot\|_{p}^{\infty}: p \in P\right\}$. Clearly, $\lambda(P) \subset \lambda^{\infty}(P)$, and the embedding is continuous. The Grothendieck-Pietsch criterion can be reformulated as follows: $\lambda(P)$ is nuclear if and only if $\lambda(P)=\lambda^{\infty}(P)$ topologically (see [20,6.1.3]). Since $P$ is assumed to be countable, this is equivalent to say that $\lambda(P)=\lambda^{\infty}(P)$ as sets (see [19, 28.16]).

EXAMPLE 5.6. $\mathbb{C}^{I}$ is nuclear. This is a standard fact (moreover, it is known that a product of nuclear spaces is nuclear), but this is also immediate from the Grothendieck-Pietsch criterion.

EXAmPLe 5.7. The Grothendieck-Pietsch criterion implies that

$$
\Lambda_{R}(\alpha) \text { is nuclear } \Leftrightarrow \begin{cases}\sup _{n}(\log n) / \alpha_{n}<\infty & \text { if } R=\infty ; \\ \lim _{n}(\log n) / \alpha_{n}=0 & \text { if } R<\infty\end{cases}
$$

(see, e.g., [19, 29.6 and 28.16]). In particular, $s$ and $\mathscr{H}\left(\mathbb{D}_{R}\right)$ are nuclear.

Definition 5.8. We say that $P$ satisfies $(\mathbf{B})$ if $P \sim P^{2}$.

The next theorem justifies the notation "(B)".

TheOREm $5.9([23,5.2])$. Let $A=\lambda(P)$ be a Köthe algebra. The following conditions are equivalent:

(i) P satisfies $(\mathbf{B})$;

(ii) $A$ is biprojective;

(iii) A is biflat;

(iv) $A$ is flat in $A$-mod;

(v) the product map $A \widehat{\otimes} A \rightarrow A, a \otimes b \mapsto a b$, is onto.

EXAmple 5.10. $\mathbb{C}^{I}$ is biprojective. Since $\mathbb{C}^{I}$ is also unital, it follows that $\mathbb{C}^{I}$ is contractible. This was first observed by Taylor [27, 5.9] (see also [10, IV.5.27]), but this is also immediate from Theorem 5.9 .

REMARK 5.11. As was shown by Helemskii [10, IV.5.27], each complete commutative contractible locally $m$-convex algebra is isomorphic to $\mathbb{C}^{I}$ for some $I$.

EXAMPLE 5.12. $\ell^{1}$ is biprojective. This fact is due to Helemskii [10, IV.5.9], but this is also immediate from Theorem 5.9 .

EXAMPLE 5.13. Theorem 5.9 implies that $\Lambda_{R}(\alpha)$ is biprojective if and only if either $R=1$ or $R=\infty$ [21, Example 3.5]. For instance, $s, \mathscr{H}(\mathbb{C}), \mathscr{H}\left(\mathbb{D}_{1}\right)$ are biprojective. Moreover, 
$\mathscr{H}\left(\mathbb{D}_{1}\right)$ is unital and hence contractible. On the other hand, $\mathscr{H}\left(\mathbb{D}_{R}\right)$ is not biprojective unless $R=1$ or $R=\infty$.

Definition 5.14. We say that $P$ satisfies $(\mathbf{M})$ if there exist complex matrices $\alpha=$ $\left(\alpha_{i j}\right)_{i, j \in I}$ and $\beta=\left(\beta_{i j}\right)_{i, j \in I}$ such that

(M1) $\alpha_{i j}+\beta_{i j}=1 \quad(i, j \in I)$;

(M2) $\forall p \in P \quad \exists C>0 \quad \exists q \in P \quad \forall j \in \mathbb{N} \quad \sup _{i}\left|\alpha_{i j}\right| p_{i} p_{j} \leq C q_{j}^{2}$;

(M3) $\forall p \in P \quad \exists C>0 \quad \exists q \in P \quad \forall i \in \mathbb{N} \quad \sup _{j}\left|\beta_{i j}\right| p_{j} p_{i} \leq C q_{i}^{2}$.

The importance of condition (M) will become clear in Section 6 . Since this condition looks a bit artificial and difficult to check, let us show that it is often satisfied automatically.

Proposition 5.15. Let $P$ be a Köthe set on $\mathbb{N}$, and suppose that either each $p \in P$ is nondecreasing, or each $p \in P$ is nonincreasing. Then $P$ satisfies $(\mathbf{M})$.

Proof. For convenience, let us agree that $a / 0=+\infty$ and $a+(+\infty)=+\infty$ for all $a \geq 0$. The advantage of this convention is that

$$
a \geq b c \Leftrightarrow a / b \geq c \text { for all } a, b, c \geq 0 .
$$

For each $i, j \in \mathbb{N}$ set

$$
\alpha_{i j}=\min \left\{1, \inf _{p \in P} \frac{p_{j}}{p_{i}}\right\} .
$$

By (3), we have

$$
\alpha_{i j} p_{i} p_{j} \leq p_{j}^{2} \quad(p \in P, i, j \in \mathbb{N}),
$$

and so $\alpha=\left(\alpha_{i j}\right)_{i, j \in \mathbb{N}}$ satisfies (M2). Now observe that the condition imposed on $P$ implies the inequality

$$
\frac{p_{i}}{p_{j}}+\frac{q_{j}}{q_{i}} \geq 1 \quad(p, q \in P, i, j \in \mathbb{N}),
$$

whence

$$
\frac{p_{i}}{p_{j}}+\alpha_{i j} \geq 1 \quad(p \in P, i, j \in \mathbb{N}) .
$$

Setting $\beta_{i j}=1-\alpha_{i j}$ and using (4) and (3), we see that

$$
\beta_{i j} p_{i} p_{j} \leq p_{i}^{2} \quad(p \in P, i, j \in \mathbb{N}),
$$

and so $\beta=\left(\beta_{i j}\right)_{i, j \in \mathbb{N}}$ satisfies (M3). Clearly, (M1) is also satisfied.

ExAmple 5.16. The Köthe sets from Examples 2.1 2.5 satisfy the conditions of Proposition 5.15, and hence they satisfy condition (M). Strictly speaking, this is not the case for $\Lambda_{R}(\alpha)$ if $R>1$, but in this case the Köthe set $P=\left\{\left(r^{\alpha_{k}}\right)_{k \in \mathbb{N}}: 0<r<R\right\}$ is equivalent to the Köthe set $P^{\prime}=\left\{\left(r^{\alpha_{k}}\right)_{k \in \mathbb{N}}: 1<r<R\right\}$, which satisfies the conditions of Proposition 5.15 .

REMARK 5.17. Condition (M) slightly differs from its original version in [21, 4.7] (see also [23, 7.2], 24, Section 3]). Namely, in [21, 23, 24, the right-hand side of (M2) (respectively, (M3)) was $C q_{j}$ (respectively, $C q_{i}$ ). We have made this change in order to prove Proposition 5.15. This will not affect our main results (Theorems 6.1 and 6.2), because for Köthe sets satisfying $(\mathbf{B})$ the old form of $(\mathbf{M})$ is clearly equivalent to the new one. 
6. Homological dimensions of Köthe algebras. In this section we compute the dimensions dg, db, w.dg, and w.db of Köthe algebras in terms of conditions $(\mathbf{U}),(\mathbf{N})$, (B), (M) introduced above. Before formulating our results, let us observe that, if $\lambda(P)$ is a Köthe algebra (i.e., if $P \prec P^{2}$ ), then $\lambda^{\infty}(P)$ is also a Fréchet algebra under pointwise multiplication (see the proof of Proposition 3.1. (iii) $\Rightarrow$ (ii)). Since $\lambda(P)$ in continuously embedded into $\lambda^{\infty}(P)$, we may consider $\lambda^{\infty}(P)$ as a left Fréhcet $\lambda(P)$-module.

Given a Fréchet algebra $A$, we consider $\mathbb{C}$ as a left Fréchet $A$-module by letting $A$ act on $\mathbb{C}$ trivially. In other words, $\mathbb{C}=A_{+} / A$.

Theorem $6.1([24,4.3])$. Let $A=\lambda(P)$ be a Köthe algebra. Then

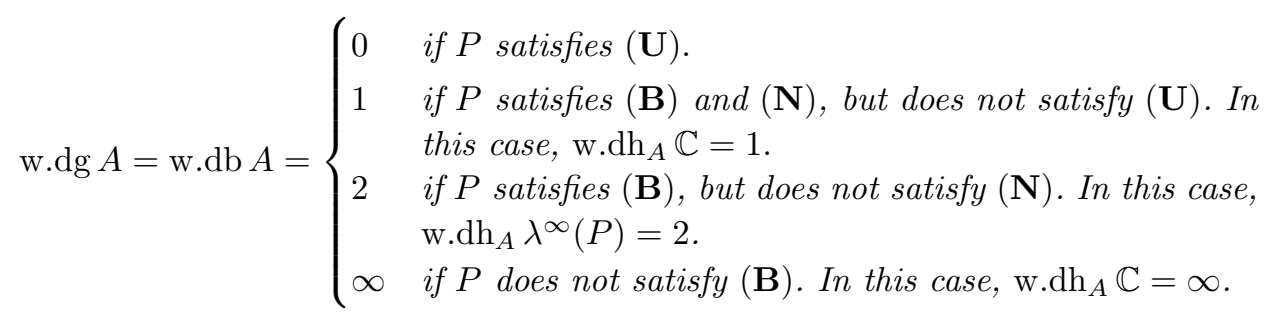

Let us note that Theorem 6.1 involves only conditions $(\mathbf{U}),(\mathbf{B})$, and $(\mathbf{N})$, but not (M). The situation with the "strong" dimensions dg and db is slightly more delicate. To compute them, we need some extra notation.

Let $P$ be a Köthe set on $I$. For each $p \in P$ we define a function $\bar{p}: I \rightarrow \mathbb{R}_{+}$by $\bar{p}_{i}=\min \left\{p_{i}, 1\right\}$. Clearly, $\bar{P}=\{\bar{p}: p \in P\}$ is a Köthe set. It is easy to show (see [24, 3.2]) that if $\lambda(P)$ is a Köthe algebra, then the pointwise product $a \cdot x$ of any $a \in \lambda(P)$ and $x \in \lambda(\bar{P})$ is in $\lambda(\bar{P})$, and that $\lambda(\bar{P})$ becomes a Fréchet $\lambda(P)$-module under this operation.

Theorem $6.2([24,4.3])$. Let $A=\lambda(P)$ be a Köthe algebra. Then

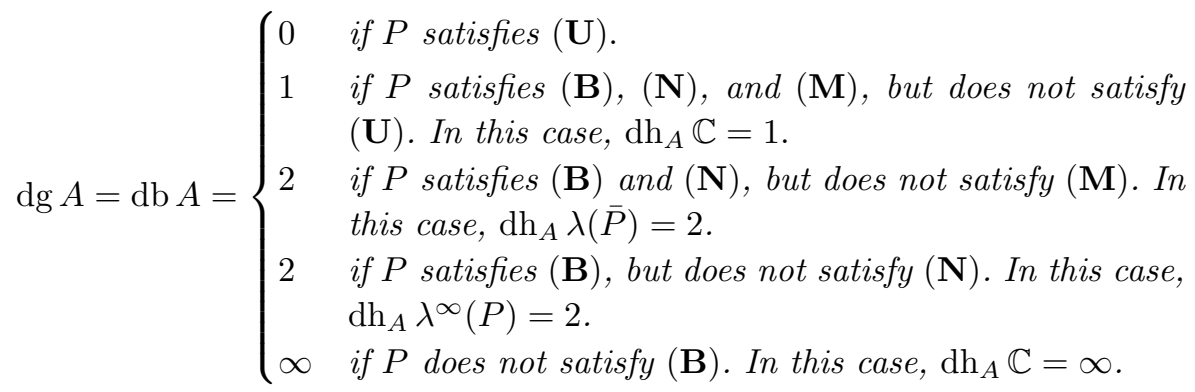

Corollary 6.3. For a Köthe algebra $\lambda(P)$, the following conditions are equivalent:

(i) $\lambda(P)$ is amenable;

(ii) $\lambda(P)$ is contractible;

(iii) $\lambda(P)$ is unital.

Corollary 6.4. Condition $(\mathbf{U})$ implies conditions $(\mathbf{B}),(\mathbf{N}),(\mathbf{M})$.

Let us look at some examples. We will see, in particular, that every combination of (U), (N), (B), (M) mentioned in Theorems 6.1 and 6.2 is possible. 
ExAmple 6.5. The algebra $\mathbb{C}^{I}$ satisfies $(\mathbf{U})$, and so

$$
\operatorname{dg} \mathbb{C}^{I}=\operatorname{db} \mathbb{C}^{I}=\text { w.dg } \mathbb{C}^{I}=\text { w.db } \mathbb{C}^{I}=0
$$

(see Example 5.10 or Corollary 6.3.

ExAmple 6.6. The algebra $\ell^{1}$ satisfies $(\mathbf{B})$, but does not satisfy $(\mathbf{N})$. Therefore Theorems 6.1 and 6.2 imply that

$$
\operatorname{dg} \ell^{1}=\operatorname{db} \ell^{1}=\mathrm{w} \cdot \operatorname{dg} \ell^{1}=\mathrm{w} \cdot \mathrm{db} \ell^{1}=\operatorname{dh}_{\ell^{1}} \ell^{\infty}=\mathrm{w} \cdot \mathrm{dh}_{\ell^{1}} \ell^{\infty}=2 .
$$

For $\mathrm{dg}, \mathrm{db}$, and $\mathrm{dh}_{\ell^{1}} \ell^{\infty}$, this is an old result by Helemskii [9] (see also [10, V.2.16]); for w.db, the result is due to $\mathrm{Yu}$. V. Selivanov [26].

EXAmple 6.7. The algebra $\Lambda_{\infty}(\alpha)$ does not satisfy (U) (see Example 5.4), but satisfies $(\mathbf{B})$ and $(\mathbf{M})$ (see Examples 5.13 and 5.16). Taking into account Example 5.7 and Theorems 6.1 and 6.2 , we get

$$
\operatorname{dg} \Lambda_{\infty}(\alpha)=\operatorname{db} \Lambda_{\infty}(\alpha)=\text { w.dg } \Lambda_{\infty}(\alpha)=\text { w.db } \Lambda_{\infty}(\alpha)= \begin{cases}1 & \text { if } \sup _{n}(\log n) / \alpha_{n}<\infty \\ 2 & \text { otherwise }\end{cases}
$$

In particular,

$$
\begin{aligned}
\operatorname{dg} s & =\operatorname{db} s=\text { w.dg } s=\text { w.db } s=1, \\
\operatorname{dg} \mathscr{H}(\mathbb{C}) & =\operatorname{db} \mathscr{H}(\mathbb{C})=\text { w.dg } \mathscr{H}(\mathbb{C})=\text { w.db } \mathscr{H}(\mathbb{C})=1 .
\end{aligned}
$$

Example 6.8. The algebra $\Lambda_{1}(\alpha)$ satisfies (B) (see Example 5.13). By using the nuclearity criterion for $\Lambda_{1}(\alpha)$ (see Example 5.7), it is easy to show that if $\Lambda_{1}(\alpha)$ satisfies (N), then it satisfies $(\mathbf{U})$. Taking into account Theorems 6.1 and 6.2 we see that

$$
\operatorname{dg} \Lambda_{1}(\alpha)=\operatorname{db} \Lambda_{1}(\alpha)=\text { w.dg } \Lambda_{1}(\alpha)=\text { w.db } \Lambda_{1}(\alpha)= \begin{cases}0 & \text { if } \lim _{n}(\log n) / \alpha_{n}<\infty \\ 2 & \text { otherwise }\end{cases}
$$

In particular,

$$
\operatorname{dg} \mathscr{H}\left(\mathbb{D}_{1}\right)=\operatorname{db} \mathscr{H}\left(\mathbb{D}_{1}\right)=\mathrm{w} \cdot \mathrm{dg} \mathscr{H}\left(\mathbb{D}_{1}\right)=\mathrm{w} \cdot \mathrm{db} \mathscr{H}\left(\mathbb{D}_{1}\right)=0 .
$$

ExAmple 6.9. If $1<R<+\infty$, then $\Lambda_{R}(\alpha)$ does not satisfy (B) (see Example 5.13). Therefore Theorems 6.1 and 6.2 imply that

$$
\operatorname{dg} \Lambda_{R}(\alpha)=\operatorname{db} \Lambda_{R}(\alpha)=\mathrm{w} \cdot \operatorname{dg} \Lambda_{R}(\alpha)=\mathrm{w} \cdot \operatorname{db} \Lambda_{R}(\alpha)=\infty .
$$

In particular,

$$
\operatorname{dg} \mathscr{H}\left(\mathbb{D}_{R}\right)=\operatorname{db} \mathscr{H}\left(\mathbb{D}_{R}\right)=\text { w.dg } \mathscr{H}\left(\mathbb{D}_{R}\right)=\mathrm{w} \cdot \operatorname{db} \mathscr{H}\left(\mathbb{D}_{R}\right)=\infty .
$$

EXAmple 6.10. Let $I=\mathbb{N} \times \mathbb{N}$. For each $i, j, k \in \mathbb{N}$ we define

$$
p_{i j}^{(k)}= \begin{cases}2^{(k j)^{i}}(i+j)^{k} & (i \leq k), \\ (i+j)^{k} & (i>k) .\end{cases}
$$

Set $p^{(k)}=\left(p_{i j}^{(k)}\right)_{i, j \in \mathbb{N}}$, and consider the Köthe set $P=\left\{p^{(k)}\right\}_{k \in \mathbb{N}}$. As was shown in [23, Theorem 7.9], $P$ satisfies $(\mathbf{B})$ and $(\mathbf{N})$, but does not satisfy $(\mathbf{M})$. Note that, since $p_{i j}^{(k)} \geq 1$ 
for all $i, j, k$, we have $\lambda(\bar{P})=\ell^{1}$. Now Theorems 6.1 and 6.2 imply that

$$
\begin{aligned}
\operatorname{dg} \lambda(P) & =\operatorname{db} \lambda(P)=\operatorname{dh}_{\lambda(P)} \ell^{1}=2, \quad \text { while } \\
\text { w.dg } \lambda(P) & =\text { w.db } \lambda(P)=\text { w.dh } \operatorname{dh}_{\lambda(P)} \mathbb{C}=1 .
\end{aligned}
$$

7. Approximate contractibility of Köthe algebras and around. Approximately contractible and approximately amenable Banach algebras were introduced by F. Ghahramani and R. J. Loy [5] and have attracted much attention in recent years [4, 6, 17, 2, 3]. Similar notions for Fréchet algebras were studied by P. Lawson and C. J. Read [18]. By definition, a Fréchet algebra $A$ is approximately contractible (respectively, approximately amenable) if for each Fréchet $A$-bimodule $X$ every continuous derivation from $A$ to $X$ (respectively, to the strong dual $X^{*}$ ) is the limit of a pointwise convergent net of inner derivations. In [6], it was shown that approximate contractibility and approximate amenability for Banach algebras are equivalent. A similar result for locally $m$-convex Fréchet algebras was proved in [18]. We refer the reader to Y. Zhang's survey [28] for a detailed discussion and numerous references concerning approximate amenability for Banach algebras.

In [18, Lawson and Read gave some sufficient conditions of approximate contractibility for locally $m$-convex Köthe algebras. In particular, they showed that the algebra $s$ of rapidly decreasing sequences is approximately contractible. The following result generalizes Proposition 3.9 from [18.

TheOrem 7.1. Let $A=\lambda(P)$ be a Köthe algebra satisfying $(\mathbf{B})$ and $(\mathbf{N})$. Then $A$ is approximately contractible.

Before proving Theorem 7.1, let us recall a result from [18].

Lemma 7.2 ([18, Lemma 2.7]). Let $A$ be a Fréchet algebra, and let $\left\{\|\cdot\|_{p}: p \in P\right\}$ be a family of seminorms generating the topology of $A$. Suppose that there exist nets $\left(u_{\lambda}\right)_{\lambda \in \Lambda}$ in $A$ and $\left(d_{\lambda}\right)_{\lambda \in \Lambda}$ in $A \widehat{\otimes} A$ such that

(i) $\left(u_{\lambda}\right)$ is a two-sided approximate identity in $A$;

(ii) $\left\|a-a u_{\lambda}\right\|_{p}\left\|u_{\lambda}\right\|_{p} \rightarrow 0$ and $\left\|a-u_{\lambda} a\right\|_{p}\left\|u_{\lambda}\right\|_{p} \rightarrow 0 \quad(a \in A)$;

(iii) $\pi\left(d_{\lambda}\right)=2 u_{\lambda}-u_{\lambda}^{2}$, where $\pi: A \widehat{\otimes} A \rightarrow A$ is the product map;

(iv) $a \cdot d_{\lambda}-d_{\lambda} \cdot a \rightarrow 0 \quad(a \in A)$.

Then $A$ is approximately contractible.

REMARK 7.3. In [18, the authors consider only locally $m$-convex Fréchet algebras. However, the argument used in [18] shows that both Lemma 7.2 and [18, Theorem 2.4], on which Lemma 7.2 is based, are true for all Fréchet algebras, not necessarily locally $m$-convex.

In the case of Köthe algebras, Lemma 7.2 can be simplified as follows.

Lemma 7.4. Let $A=\lambda(P)$ be a Köthe algebra, and let $\Pi \subset A$ denote the set of characteristic functions $\chi_{J}$, where $J$ runs over the family of all finite subsets of $I$. Suppose that 
for each $p \in P$ and each $a \in A$ there exists $u \in \Pi$ such that

$$
\begin{gathered}
\|a-a u\|_{p}<1, \\
\|a-a u\|_{p}\|u\|_{p}<1 .
\end{gathered}
$$

Then $A$ is approximately contractible.

Proof. Let $\mathscr{F}$ denote the family of all finite subsets of $A$, and let

$$
\Lambda=\{(F, p, \varepsilon): F \in \mathscr{F}, p \in P, \varepsilon>0\} .
$$

We make $\Lambda$ into a directed poset by setting

$$
(F, p, \varepsilon) \preceq\left(F^{\prime}, p^{\prime}, \varepsilon^{\prime}\right) \quad \Leftrightarrow \quad F \subset F^{\prime}, p \leq p^{\prime}, \varepsilon \geq \varepsilon^{\prime} .
$$

Fix any $\lambda=(F, p, \varepsilon) \in \Lambda$, and define $b \in A$ by

$$
b=\varepsilon^{-1} \sum_{a \in F}|a| .
$$

Using (5) and (6), find $u_{\lambda}=\chi_{J} \in \Pi$ such that

$$
\left\|b-b u_{\lambda}\right\|_{p}<1, \quad\left\|b-b u_{\lambda}\right\|\left\|_{p}\right\| u_{\lambda} \|_{p}<1 .
$$

Since

$$
\|a-a u\|_{p}=\sum_{i \in I \backslash J}\left|a_{i}\right| p_{i}
$$

it follows that

$$
\begin{array}{cc}
\left\|a-a u_{\lambda}\right\|_{p}<\varepsilon & (a \in F), \\
\left\|a-a u_{\lambda}\right\|_{p}\left\|u_{\lambda}\right\|_{p}<\varepsilon & (a \in F) .
\end{array}
$$

Therefore the net $\left(u_{\lambda}\right)_{\lambda \in \Lambda}$ satisfies conditions (i) and (ii) of Lemma 7.2. Now, following [18, Example 3.1], define $d_{\lambda} \in A \widehat{\otimes} A$ by

$$
d_{\lambda}=\sum_{i \in F} e_{i} \otimes e_{i},
$$

where $e_{i}$ stands for the function on $I$ which is 1 at $i, 0$ elsewhere. Then it is clear that

$$
\begin{gathered}
\pi\left(d_{\lambda}\right)=u_{\lambda}=2 u_{\lambda}-u_{\lambda}^{2} ; \\
a \cdot d_{\lambda}=d_{\lambda} \cdot a \quad(a \in A) .
\end{gathered}
$$

Thus conditions (iii) and (iv) of Lemma 7.2 are also satisfied, and so $A$ is approximately contractible.

Proof of Theorem 7.1. Fix any $a \in A$ and $p \in P$, and find $q \in P$ and $\lambda \in \ell^{1}(I)$ such that $p \leq q$ and $p \leq \lambda q$. Let

$$
I^{\prime}=\left\{i \in I: p_{i}>1\right\}, \quad I^{\prime \prime}=\left\{i \in I: p_{i} \leq 1\right\} .
$$

Note that $\lambda_{i}>0$ whenever $i \in I^{\prime}$. For each $n \in \mathbb{N}$, define the subset $J_{n}^{\prime} \subset I^{\prime}$ as follows. If $I^{\prime}$ is finite, we set $J_{n}^{\prime}=I^{\prime}$. Otherwise, let

$$
J_{n}^{\prime}=\left\{i \in I^{\prime}: q_{i} \leq n\right\} .
$$

For each $i \in I^{\prime}$ we have

$$
q_{i} \geq p_{i} / \lambda_{i}>1 / \lambda_{i} \rightarrow \infty \quad\left(i \in I^{\prime}, i \rightarrow \infty\right) .
$$

Therefore $J_{n}^{\prime}$ is finite. 
Choose a finite subset $J_{n}^{\prime \prime} \subset I^{\prime \prime}$ such that

$$
\sup _{i \in I^{\prime \prime} \backslash J_{n}^{\prime \prime}}\left|a_{i}\right| p_{i}<1 / n^{2} .
$$

Finally, let $J_{n}=J_{n}^{\prime} \sqcup J_{n}^{\prime \prime}$ and $u_{n}=\chi_{J_{n}}$. Since $P$ satisfies $(\mathbf{N})$, we have $\lambda(P)=\lambda^{\infty}(P)$ topologically (see the discussion after Definition 5.5), and so the topology on $\lambda(P)$ is determined by the seminorms $\|\cdot\|_{p}^{\infty}(p \in P)$. Therefore by Lemma 7.4 it suffices to show that

$$
\left\|a-a u_{n}\right\|_{p}^{\infty}\left(1+\left\|u_{n}\right\|_{p}^{\infty}\right) \rightarrow 0 \quad(n \rightarrow \infty) .
$$

Since

$$
\left\|u_{n}\right\|_{p}^{\infty}=\sup _{j \in J_{n}} p_{j} \leq n
$$

we have

$$
\left\|a-a u_{n}\right\|_{p}^{\infty}\left(1+\left\|u_{n}\right\|_{p}^{\infty}\right) \leq 2 n\left\|a-a u_{n}\right\|_{p}^{\infty},
$$

and it remains to show that

$$
n\left\|a-a u_{n}\right\|_{p}^{\infty} \rightarrow 0 \quad(n \rightarrow \infty) .
$$

Observe that

$$
n\left\|a-a u_{n}\right\|_{p}^{\infty}=\sup _{i \in I \backslash J_{n}}\left|a_{i}\right| p_{i} n=\max \left\{\sup _{i \in I^{\prime} \backslash J_{n}^{\prime}}\left|a_{i}\right| p_{i} n, \sup _{i \in I^{\prime \prime} \backslash J_{n}^{\prime \prime}}\left|a_{i}\right| p_{i} n\right\} .
$$

By (7), we have

$$
\sup _{i \in I^{\prime \prime} \backslash J_{n}^{\prime \prime}}\left|a_{i}\right| p_{i} n \leq 1 / n \rightarrow 0 \quad(n \rightarrow \infty) .
$$

For each $i \in I^{\prime} \backslash J_{n}^{\prime}$ we have $q_{i}>n$. Therefore

$$
\sup _{i \in I^{\prime} \backslash J_{n}^{\prime}}\left|a_{i}\right| p_{i} n \leq \sup _{i \in I^{\prime} \backslash J_{n}^{\prime}}\left|a_{i}\right| q_{i}^{2} \rightarrow 0 \quad(n \rightarrow \infty),
$$

because $P$ satisfies (B). Now (8) follows from (9), (10), and (11).

EXAMPLE 7.5. Theorem 7.1 implies that $\Lambda_{\infty}(\alpha)$ is approximately contractible whenever $\sup _{n}(\log n) / \alpha_{n}<\infty($ see Examples 5.7 and 5.13$)$. In particular, $s$ and $\mathscr{H}(\mathbb{C})$ are approximately contractible.

EXAMPLE 7.6. As was shown in [4, $\ell^{1}$ is not approximately contractible (although it satisfies (B), see Example 5.12.

Let us now discuss some other properties of Köthe algebras related to approximate contractibility. One such property is idempotence. Given an algebra $A$, let

$$
A^{2}=\operatorname{span}\{a b: a, b \in A\} .
$$

In [4, the authors conjectured that there is a relation between the approximate contractibility of a Banach sequence algebra $A$ and the property $A=A^{2}$. Below we study this relation in the case of Köthe algebras.

Lemma 7.7. Let $A=\lambda(P)$ be a Köthe algebra. Then

$$
A^{2}=\left\{a^{2}: a \in A\right\}=\{a \in A: \sqrt{|a|} \in A\} .
$$


Proof. Suppose that $\sqrt{|a|} \in A$. For each $i \in I$, find $\lambda_{i} \in \mathbb{C}$ such that $a_{i}=\lambda_{i}^{2}\left|a_{i}\right|$, and define $b_{i}=\lambda_{i} \sqrt{\left|a_{i}\right|}$. We then have $b=\left(b_{i}\right) \in A$ and $a=b^{2}$. Clearly, this implies that $a \in A^{2}$.

Conversely, suppose that $a \in A^{2}$, and write

$$
a=\sum_{k=1}^{n} b_{k} c_{k} \quad\left(b_{k}, c_{k} \in A\right) .
$$

For each $p \in P$ and each $i \in I$ we have

$$
\left|a_{i}\right| p_{i}^{2} \leq \sum_{k}\left|b_{k i}\right|\left|c_{k i}\right| p_{i}^{2} \leq\left(\sum_{k}\left(\left|b_{k i}\right| p_{i}+\left|c_{k i}\right| p_{i}\right)\right)^{2} .
$$

Therefore

$$
\sum_{i} \sqrt{\left|a_{i}\right|} p_{i} \leq \sum_{i} \sum_{k}\left(\left|b_{k i}\right| p_{i}+\left|c_{k i}\right| p_{i}\right)=\sum_{k}\left(\left\|b_{k}\right\|_{p}+\left\|c_{k}\right\|_{p}\right)<\infty,
$$

which implies that $\sqrt{|a|} \in A$.

REMARK 7.8. An inspection of the above proof shows that, if $A$ is a Köthe algebra over $\mathbb{R}$, then

$$
A^{2}=\{a b: a, b \in A\}=\{a \in A: \sqrt{|a|} \in A\} .
$$

Let $\lambda(P)$ be a Köthe space. Recall that the Köthe-Toeplitz dual $\lambda(P)^{\times}$is defined by

$$
\lambda(P)^{\times}=\left\{y=\left(y_{i}\right) \in \mathbb{C}^{I}:\|y\|_{x}=\sum_{i}\left|y_{i} x_{i}\right|<\infty \quad \forall x \in \lambda(P)\right\} .
$$

In other words, $\lambda(P)^{\times}$is the Köthe space $\lambda\left(P^{\times}\right)$, where $P^{\times}=\left\{\left(\left|x_{i}\right|\right): x \in \lambda(P)\right\}$.

Lemma 7.9. Let $A=\lambda(P)$ be a Köthe algebra satisfying $(\mathbf{B})$. Suppose that $A^{\times}$is nuclear. Then $A=A^{2}$.

Proof. Fix any $a \in A$. Since $A^{\times}=\lambda\left(P^{\times}\right)$is nuclear, the Grothendieck-Pietsch criterion implies that $P^{\times}$satisfies (N). Therefore there exist $\lambda \in \ell^{1}$ and $b \in P^{\times}$such that $|a| \leq \lambda b$. For the same reason, there exist $\mu \in \ell^{1}$ and $c \in P^{\times}$such that $b \leq \mu c$. Now take any $p \in P$ and find $q \in P$ and $C>0$ such that $p^{2} \leq C^{2} q$. We have

$$
\sum_{i} \sqrt{\left|a_{i}\right|} p_{i} \leq C \sum_{i} \sqrt{\left|a_{i}\right| q_{i}} \leq C \sum_{i} \sqrt{\lambda_{i} \mu_{i} c_{i} q_{i}} \leq C \sup _{i} \sqrt{c_{i} q_{i}} \sum_{i} \sqrt{\lambda_{i} \mu_{i}} .
$$

Since $\lambda, \mu \in \ell^{1}$, we have $\sqrt{\lambda}, \sqrt{\mu} \in \ell^{2}$, and so $\sum_{i} \sqrt{\lambda_{i} \mu_{i}}<\infty$. Since $c \in A$, we have $\sup _{i} \sqrt{c_{i} q_{i}} \leq \sqrt{\|c\|_{q}}<\infty$. Now 12 implies that $\sum_{i} \sqrt{\left|a_{i}\right|} p_{i}<\infty$, i.e., $\sqrt{|a|} \in A$. Using Lemma 7.7. we see that $a \in A^{2}$, and so $A=A^{2}$, as claimed.

Lemma 7.10. Let $A=\lambda(P)$ be a Köthe algebra on $\mathbb{N}$ satisfying $(\mathbf{B})$. Suppose that $p_{n} \geq 1$ for all $p \in P$ and all $n \in \mathbb{N}$, and suppose that there exists $p \in P$ such that

$$
\sup _{n} \frac{\log n}{\log p_{n}}<\infty .
$$

Then $A$ is nuclear.

Proof. Since $P$ is directed (Axiom (P2)), the topology on $\lambda(P)$ is generated by all seminorms $\|\cdot\|_{q}$ with $q \in P, q \geq p$. Therefore we may assume that $(13)$ holds for all $p \in P$. 
Now fix any $p \in P$ and find $k \in \mathbb{N}$ such that $\log n \leq k \log p_{n}$ for all $n \in \mathbb{N}$. Since $P$ satisfies (B), there exist $C>0$ and $q \in P$ such that $p^{2 k+1} \leq C q$. We have

$$
\sum_{n} \frac{p_{n}}{q_{n}} \leq C \sum_{n} \frac{1}{p_{n}^{2 k}} \leq C \sum_{n} \frac{1}{n^{2}}<\infty .
$$

Now the Grothendieck-Pietsch criterion implies that $A$ is nuclear.

Lemma 7.11. Let $A=\lambda(P)$ be a Köthe algebra on $\mathbb{N}$ such that $A=A^{2}$. Suppose that $1 \leq p_{n} \leq p_{n+1}$ for all $p \in P$ and all $n \in \mathbb{N}$. Then there exists $p \in P$ such that (13) holds.

Proof. Without loss of generality, we may assume that $P=\left\{p^{(n)}: n \in \mathbb{N}\right\}$, and that $p^{(n)} \leq p^{(n+1)}$ for all $n$. Assume, towards a contradiction, that none of $p \in P$ satisfies (13). Then for each $n \in \mathbb{N}$ there exists $k_{n} \in \mathbb{N}$ such that

$$
\frac{\log k_{n}}{\log p_{k_{n}}^{(n)}} \geq n, \quad \text { i.e., } p_{k_{n}}^{(n)} \leq k_{n}^{1 / n} .
$$

We may also assume that $k_{n+1} \geq 2 k_{n}$ for all $n \in \mathbb{N}$. Set $k_{0}=0$ and define $a \in \mathbb{C}^{\mathbb{N}}$ by

$$
a_{m}=\frac{1}{k_{n}^{3}} \quad \text { for } k_{n-1}<m \leq k_{n} .
$$

Then for each $i \in \mathbb{N}$ we have

$$
\begin{aligned}
\sum_{m>k_{i-1}}\left|a_{m}\right| p_{m}^{(i)} & =\sum_{n \geq i} \sum_{m=k_{n-1}+1}^{k_{n}}\left|a_{m}\right| p_{m}^{(i)} \leq \sum_{n \geq i} \sum_{m=k_{n-1}+1}^{k_{n}} \frac{1}{k_{n}^{3}} p_{k_{n}}^{(n)} \\
& \leq \sum_{n \geq i} \sum_{m=k_{n-1}+1}^{k_{n}} k_{n}^{\frac{1}{n}-3}=\sum_{n \geq i}\left(k_{n}-k_{n-1}\right) k_{n}^{\frac{1}{n}-3} \leq \sum_{n \geq i} k_{n}^{\frac{1}{n}-2}<\infty
\end{aligned}
$$

because $\frac{1}{n}-2<-\frac{3}{2}$ for $n \geq 2$. Therefore $a \in A$. On the other hand,

$$
\sum_{m} \sqrt[4]{\left|a_{m}\right|}=\sum_{n} \sum_{m=k_{n-1}+1}^{k_{n}} \frac{1}{k_{n}^{3 / 4}} \geq \sum_{n} \frac{k_{n}-k_{n-1}}{k_{n}} \geq \sum_{n} \frac{1}{2}=\infty,
$$

showing that $\sqrt[4]{|a|} \notin \ell^{1}$, and, a fortiori, $\sqrt[4]{|a|} \notin A$. Applying Lemma 7.7 twice, we see that $a \notin A$. The resulting contradiction completes the proof.

Now we can summarize the above discussion as follows.

THEOREM 7.12. Let $A=\lambda(P)$ be a Köthe algebra on $\mathbb{N}$ satisfying $(\mathbf{B})$. Suppose that $1 \leq p_{n} \leq p_{n+1}$ for all $p \in P$ and all $n \in \mathbb{N}$. Then the following conditions are equivalent:

(i) $A$ is nuclear;

(ii) $A^{\times}$is nuclear;

(iii) $A=A^{2}$;

(iv) there exists $p \in P$ such that

$$
\sup _{n} \frac{\log n}{\log p_{n}}<\infty .
$$

Each of the above conditions implies that $A$ is approximately contractible.

Proof. (i) $\Rightarrow$ (ii). This is true for all metrizable Köthe spaces [16, Satz 7].

(ii) $\Rightarrow$ (iii). This follows from Lemma 7.9 . 
(iii) $\Rightarrow$ (iv). This follows from Lemma 7.11

(iv) $\Rightarrow$ (i). This follows from Lemma 7.10 .

Finally, (i) implies that $A$ is approximately contractible by Theorem 7.1 .

8. Open problems. In conclusion, let us formulate some open problems.

Problem 8.1. Let $A$ be a biflat Fréchet algebra. Is w.db $A \leq 2$ ?

Recall that the answer is positive if $A$ is a Banach algebra [12, 2.5.8] or if $A$ is projective in $A$-mod (or in mod- $A$ ) [23, Proposition 4.8].

In fact, Problem 8.1 can be reduced to the following.

Problem 8.2. Let $X$ be flat in $A$-mod, and let $Y$ be flat in $\bmod -A$. Is $X \widehat{\otimes} Y$ flat in $A$-mod- $A$ ?

Again, the answer is positive in the case of Banach modules over Banach algebras [11, 7.1.57]. The answer is also positive provided that either $X$ or $Y$ is projective [23. Proposition 3.6].

Problem 8.3. Let $A=\lambda(P)$ be an approximately contractible Köthe algebra.

(1) Does $P$ satisfy $(\mathbf{B})$ ?

(2) Does $A$ satisfy the conditions (i)-(iv) of Theorem 7.12? In particular, must $A$ be nuclear?

Note that, if $A=A^{2}$, then $P$ satisfies (B) (see implication (v) $\Rightarrow$ (i) in Theorem 5.9). Therefore a positive answer to question (2) would imply a positive answer to question (1).

Acknowledgments. This paper is based on a lecture delivered at the $19^{\text {th }}$ International Conference on Banach Algebras held at Będlewo, July 14-24, 2009. The support for the meeting by the Polish Academy of Sciences, the European Science Foundation under the ESF-EMS-ERCOM partnership, and the Faculty of Mathematics and Computer Science of the Adam Mickiewicz University at Poznań is gratefully acknowledged. The author was also supported by the RFBR grant 08-01-00867, by the Ministry of Education and Science of Russia (programme "Development of the scientific potential of the Higher School", grant no. 2.1.1/2775), and by the President of Russia grant MK-1173.2009.1.

The author is grateful to C. J. Read for explaining some points of [18].

\section{References}

[1] S. J. Bhatt and G. M. Deheri, Köthe spaces and topological algebras with bases, Proc. Indian Acad. Sci. Math. Sci. 100 (1990), 259-273.

[2] Y. Choi, F. Ghahramani, and Y. Zhang, Approximate and pseudo-amenability of various classes of Banach algebras, J. Funct. Anal. 256 (2009), 3158-3191.

[3] Y. Choi and F. Ghahramani, Approximate amenability of Schatten classes, Lipschitz algebras and second duals of Fourier algebras, Q. J. Math., to appear (2010).

[4] H. G. Dales, R. J. Loy, and Y. Zhang, Approximate amenability for Banach sequence algebras, Studia Math. 177 (2006), 81-96. 
[5] F. Ghahramani and R. J. Loy, Generalized notions of amenability, J. Funct. Anal. 208 (2004), 229-260.

[6] F. Ghahramani, R. J. Loy, and Y. Zhang, Generalized notions of amenability. II, J. Funct. Anal. 254 (2008), 1776-1810.

[7] F. Ghahramani and R. Stokke, Approximate and pseudo-amenability of the Fourier algebra, Indiana Univ. Math. J. 56 (2007), 909-930.

[8] A. Ya. Helemskii, The homological dimension of normed modules over Banach algebras, Mat. Sb. (N.S.) 81 (123) (1970), 430-444 (in Russian); English transl.: Mathematics of the USSR-Sbornik 10 (1970), 399-411.

[9] A. Ya. Helemskii, The global dimension of a Banach function algebra is different from one, Funkcional. Anal. i Prilozen. 6 (1972), no. 2, 95-96 (in Russian); English transl.: Functional Anal. Appl. 6 (1972), 166-168.

[10] A. Ya. Helemskii, The Homology of Banach and Topological Algebras, Moscow University Press, 1986 (in Russian); English transl.: Kluwer Academic Publishers, Dordrecht, 1989.

[11] A. Ya. Helemskii, Banach and Polynormed Algebras: General Theory, Representations, Homology, Nauka, Moscow, 1989 (in Russian); English transl.: Oxford University Press, 1993.

[12] A. Ya. Helemskii, Homology for the Algebras of Analysis, in: Handbook of Algebra, Vol. 2 (ed. M. Hazewinkel), 151-274, Amsterdam, North-Holland, 2000.

[13] B. E. Johnson, Cohomology in Banach algebras, Mem. Amer. Math. Soc. 127 (1972).

[14] R. Kiehl and J. L. Verdier, Ein einfacher Beweis des Kohärenzsatzes von Grauert, Math. Ann. 195 (1971), 24-50.

[15] G. Köthe, Die Stufenräume, eine einfache Klasse linearer vollkommener Räume, Math. Z. 51 (1948), 317-345.

[16] G. Köthe, Über nukleare Folgenräume, Studia Math. 31 (1968), 267-271.

[17] G. Köthe, Topological Vector Spaces I, Springer-Verlag, New York, 1969.

[18] P. Lawson and C. J. Read, Approximate amenability of Fréchet algebras, Math. Proc. Cambridge Philos. Soc. 145 (2008), 403-418.

[19] R. Meise and D. Vogt, Introduction to Functional Analysis, Clarendon Press, Oxford, 1997.

[20] A. Pietsch, Nuclear locally convex spaces, Springer, New York, 1972.

[21] A. Yu. Pirkovskii, Biprojective topological algebras of homological bidimension 1, J. Math. Sci. (New York) 111 (2002), 3476-3495.

[22] A. Yu. Pirkovskii, Homological bidimension of biprojective topological algebras and nuclearity, in: Proc. of the 3rd International Conference on Topological Algebra and Applications, Oulu University, Oulu, Finland, July 2-6, 2001; Acta Universitatis Ouluensis A 408 (2004), 179-196.

[23] A. Yu. Pirkovskii, Weak homological dimensions and biflat Köthe algebras, Mat. Sb. 199 (2008), no. 5, 45-80 (in Russian); English transl.: Sbornik Math. 199 (2008), no. 5, 673705.

[24] A. Yu. Pirkovskii, Homological dimensions of Köthe algebras, Q. J. Math, to appear (2010).

[25] H. Render and A. Sauer, Algebras of holomorphic functions with Hadamard multiplication, Studia Math. 118 (1996), 77-100.

[26] Yu. V. Selivanov, Weak homological bidimension and its values in the class of biflat Banach algebras, Extracta Math. 11 (1996), 348-365.

[27] J. L. Taylor, Homology and cohomology for topological algebras, Adv. Math. 9 (1972), $137-182$. 
[28] Y. Zhang, Solved and unsolved problems on generalized notions of amenability for Banach algebras, Slides of a lecture delivered at the $19^{\text {th }}$ International Conference on Banach Algebras, http://www.siue.edu/MATH/BA2009/ABSTRACTS/lecture-Zhang.pdf. 